\title{
ChemSpectra: a web-based spectra editor for analytical data
}

\author{
Yu-Chieh Huang ${ }^{1}$, Pierre Tremouilhac ${ }^{1}$, An Nguyen ${ }^{1}$, Nicole Jung ${ }^{1,2^{*}}$ (D) and Stefan Bräse ${ }^{1,2^{*}}$
}

\begin{abstract}
ChemSpectra, a web-based software to visualize and analyze spectroscopic data, integrating solutions for infrared spectroscopy (IR), mass spectrometry (MS), and one-dimensional ${ }^{1} \mathrm{H}$ and ${ }^{13} \mathrm{C}$ NMR (proton and carbon nuclear magnetic resonance) spectroscopy, is described. ChemSpectra serves as web-based tool for the analysis of the most often used types of one-dimensional spectroscopic data in synthetic (organic) chemistry research. It was developed to support in particular processes for the use of open file formats which enable the work according to the FAIR data principles. The software can deal with the open file formats JCAMP-DX (IR, MS, NMR) and mzML (MS) proposing these data file types to gain interoperable data. ChemSpectra can be extended to read also other formats as exemplified by selected proprietary mass spectrometry data files of type RAW and NMR spectra files of type FID. The JavaScriptbased editor can be integrated with other software, as demonstrated by integration into the Chemotion electronic lab notebook (ELN) and Chemotion repository, demonstrating the implementation into a digital work environment that offers additional functionality and sustainable research data management options. ChemSpectra supports different functions for working with spectroscopic data such as zoom functions, peak picking and automatic peak detection according to a default or manually defined threshold. NMR specific functions include the definition of a reference signal, the integration of signals, coupling constant calculation and multiplicity assignment. Embedded into a web application such as an ELN or a repository, the editor can also be used to generate an association of spectra to a sample and a file management. The file management supports the storage of the original spectra along with the last edited version and an automatically generated image of the spectra in png format. To maximize the benefit of the spectra editor for e.g. ELN users, an automated procedure for the transfer of the detected or manually chosen signals to the ELN was implemented. ChemSpectra is released under the AGPL license to encourage its re-use and further developments by the community.
\end{abstract}

Keywords: Spectroscopy, Analysis, NMR, IR, Mass spectrometry, JCAMP-DX

\section{Background}

The online and interactive visualization of spectroscopic data is crucial for modern scientific work to be able to evaluate scientific data and to analyze it. Web-based solutions are beneficial because of their platform-independent use and few system requirements. As web-based

\footnotetext{
*Correspondence: nicole.jung@kit.edu; stefan.braese@kit.edu 1 Institute of Biological and Chemical Systems-Functional Molecular Systems (IBCS-FMS), Karlsruhe Institute of Technology, Hermann-von-Helmholtz-Platz 1, 76344 Eggenstein-Leopoldshafen, Germany

Full list of author information is available at the end of the article
}

software developments usually can be embedded into manifold projects, they may serve as a valuable contribution to existing databases and information systems (like electronic lab notebooks, ELNs, or repositories). In chemistry research, in particular the information from ${ }^{1} \mathrm{H}$ and ${ }^{13} \mathrm{C}$ NMR, IR, and mass spectroscopic experiments is of high importance as these four techniques are essential for the identification of molecules. Due to their significance, they generally form the standard set of analytical data that has to be provided along with the scientific publication of synthetic results. NMR, MS and IR data can be analyzed either manually from printed 
spectra or they can be analyzed in detail using commercial software or free stand-alone tools. The available commercial or non-open source software products usually include manifold functionalities for processing, visualization, analysis and documentation but they have to be installed and work in most of the cases in a nonembedded manner. Examples for such software options are MestreNova [1], ChemAxon [2], TopSpin (NMR) [3] or Spectragryph (IR) [4]. Besides professional tools as the given ones, only a few web-based developments are available as an Open Source. For the spectra types NMR, MS and IR which are considered in this work, the web-based visualization tools JSpecView [5, 6], NMRPro [7], MetaboAnalyst [8], MetaboHunter [9], COLMAR [10], jsNMR [11], and SpeckTackle [12] are known. Some of them are already integrated into web services, e.g. SpeckTackle is used in MetaboLights for NMR/MS data [13]. Other databases such as the databases Human Metabolome Database HMDB [14] or DrugBank [15] are supported by additional editors that are developed internally explicitly for those databases [16].

Due to the need for advanced spectra editors for the visualization but also for the analysis of spectroscopic data such as NMR, MS and IR data with peak-picking, NMR signal integration, coupling constant calculation and multiplicity assignment, we initiated a project that is based on currently available source code and tools from own developments. The aim of the development is the extension of the applicability of web-based editors to enable their use for enhanced data management tasks in particular for web-based data management systems such as ELNs and repositories.

\section{Implementation}

ChemSpectra consists of three modules, the react-spectra-editor, chem-spectra-client, and chem-spectra-app. For the client-side implementations (react-spectra-editor and chem-spectra-client) the software is written in the programming language JavaScript using the framework React.js which offers a user-friendly single page application to upload, read, edit, and download spectra. JavaScript was chosen due to its benefits for interactive web tools to facilitate the embedding of the developments into other web-based software. The jcampconverter library [17] inside the react-spectra-editor extracts spectroscopic data of the composed JCAMP-DX file which is sent from the server-side.

The server-side spectra handling (chem-spectra-app) is based on python and was built using a modified version of NMRglue [18] and SciPy [19]. Python as a backend programming language on the framework Flask provides data processing and ensures the compatibility with and re-use of the previously developed systems.
ChemSpectra can be used as stand-alone software to be offered as an independent web service or can be used for other web developments. The stand-alone application supports the visualization and analysis of spectra, but its functions are limited in comparison to an embedded version, as the information stored in the browser exists temporarily and is not persisted permanently. To show the advantages of the ChemSpectra development as an embedded application offering the full available functionality, it was incorporated into the web applications Chemotion ELN and repository, which are developments of our research group reported earlier [20,21]. Examples for further functions are the storage and management of the files that were edited by ChemSpectra. Figure 1 gives an overview of the main parts of the ChemSpectra software and the implemented processes for the stand-alone version (green arrows) and the exemplary implementation with the Chemotion applications (workflow given in blue). While, for the stand-alone implementation, the chem-spectra-app communicates directly with the chem-spectra-client, the communication for the embedded software is managed by the server of the ELN or repository. If ChemSpectra is embedded into other web applications, further systems and work processes such as a data provider can be added to the overall workflow. In the herein depicted example, an instrument server that provides analytical data that were collected by a data collector [22] of the ELN is connected.

ChemSpectra has been optimized and was tested thoroughly on the Browser Chrome. A stand-alone server version and a version of ChemSpectra embedded into the Chemotion ELN and repository are available for demonstration at the Chemotion project website, www.chemo tion.net. In addition, the source code of the project for chem-spectra-app [23], chem-spectra-client [24], and react-spectra-editor [25] can be retrieved from github. The source code is released as an Open Source under the license AGPL version 3.

\section{Results}

The main part of the ChemSpectra software is the reactspectra-editor which displays the three types NMR, MS and IR data. The type of spectra is extracted automatically from the provided files. Depending on the extracted type, one of two layouts available for visualization is used: the line plot (NMR and IR data) or the bar graph (MS data). To edit the provided data with ChemSpectra, a control panel offers generic and data-type specific actions to analyze and configure the given data (Figs. 2 and 3). The generic actions are available for NMR, IR and MS data and allow to (1) zoom in and out (2) adjust the threshold that is given as default for each spectra type, and (3) extract the peaks and write them in a list form. 


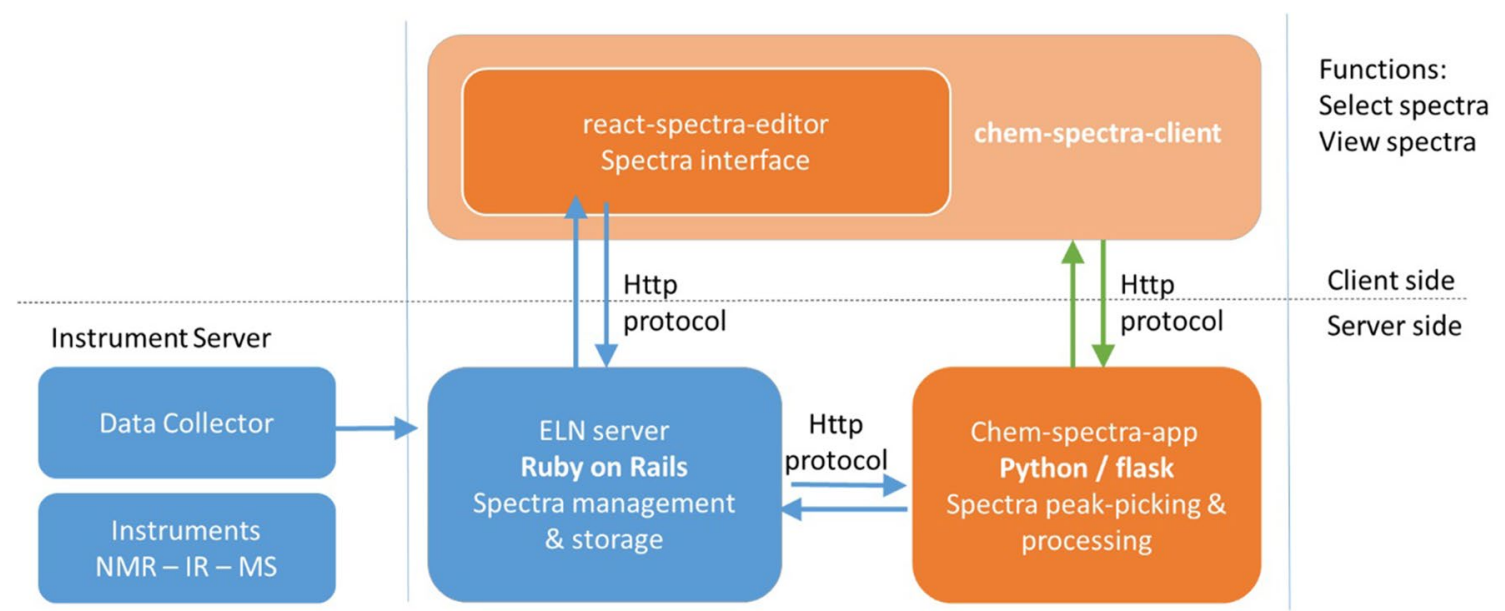

Fig. 1 General scheme describing the overall implementation. Orange=necessary parts for a stand-alone version of ChemSpectra, blue: implementation to Chemotion ELN and connection of analysis results via an instrument server

With respect to the selected signals in the spectra, the user can select the number of displayed digits for each signal and in which order the signals should be summarized (descending or ascending).

The backend part of the ChemSpectra software, the chem-spectra-app, manages the decoding and composing of spectra files, the peak-picking, and the image generation as basic parts for the transformation of the given spectra. Currently, the chem-spectra-app accepts the file extensions jcamp, jdx, and dx for NMR, IR and MS spectra, mzML [26] and RAW files for MS spectra and FID or ZIP files for NMR spectra. If ChemSpectra is integrated into a work environment such as an ELN or repository server, the chem-spectra-app is a microservice that is in charge of all spectra-related processes, excluding storage and management (which are the main requests gated by the host web application).

\section{Spectra editor and control panel for one dimensional ${ }^{1} \mathrm{H}$ NMR and ${ }^{13} \mathrm{C}$ NMR data}

As the different analysis types need different actions to edit the corresponding data, ChemSpectra enables analysis-specific actions in the react-spectra-editor UI. In the case of NMR data, these specific actions are the addition and/or removal of peaks, integration of signals, coupling constant and multiplicity calculation and assignment. Multiplicities are automatically inferred by known libraries $[27,28]$ and are checked by additional rules to ensure the correctness of the results. The generated information such as the identified signals, coupling constants and multiplicity can be summarized in form of a signal list. Additionally, the ChemSpectra editor offers a list of the most common reference solvent shifts for ${ }^{1} \mathrm{H}$ and ${ }^{13} \mathrm{C}$
NMR spectra, allowing the correction of the values given by default.

\section{Main and control panel for IR data}

The IR editor and control panel offer the general three functions given for all types of spectra: adding and removing peaks, including an overview of the added and removed signals, and additionally an option to extract the given signals. Corresponding to the reporting standards for IR spectroscopy, the intensity of the identified signals (vw, w, m, s, vs) can be added to the wavenumber that is recorded. The current implementation gives the information in brackets after the corresponding wavenumber (see Fig. 4 for IR spectra in the stand-alone software).

\section{Main and control panel for mass data}

Mass spectra differ from NMR and IR spectra in that way, that they may consist of different scans for one measurement. Depending on the internal procedure of an institution, mass spectra-if they are provided digitally-are either provided as original files including all scans that were measured, or are provided as one preselected scan. The ChemSpectra control panel for mass spectrometry offers therefore a dropdown menu including a list of the scans that are provided with each file. Per default, the first scan is visualized by the editor, but the user can change this setting to any scan that is more suitable for the analysis. Figure 3 illustrates the functions of ChemSpectra for mass spectra with the example 4-oxo- $4 H$-chromene3 -carbaldehyde. The threshold line can be used to select the signals and the unselected peaks become grey to be clearly distinguishable from the selected ones. Individual signals can be selected to show the $\mathrm{m} / \mathrm{z}$ value 


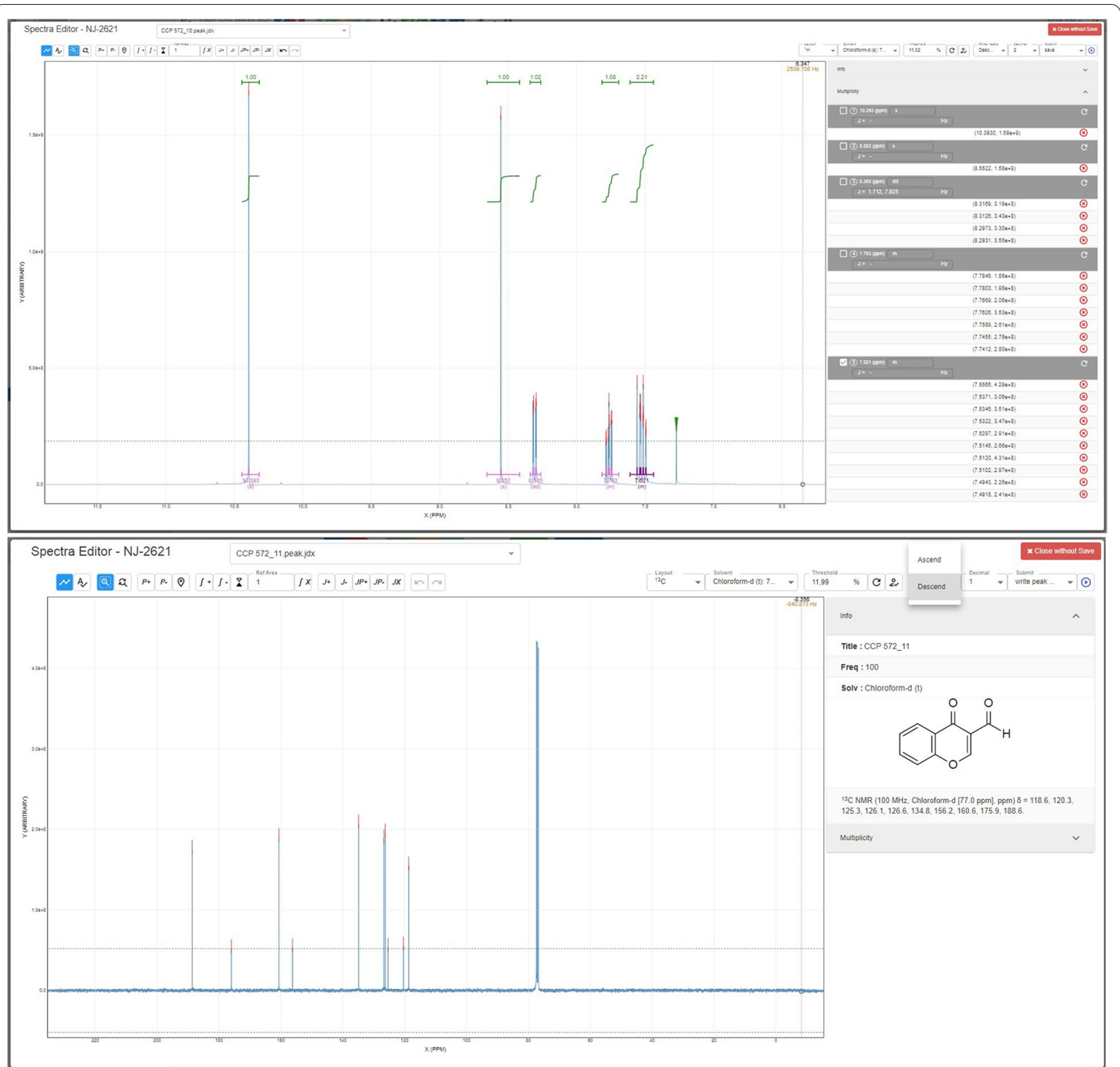

Fig. 2 UI of the react-spectra-editor for ${ }^{1} \mathrm{H}$ NMR data (upper part) and ${ }^{13} \mathrm{C}$ NMR data (lower part) generated for a commercial sample of 4-oxo-4H-chromene-3-carbaldehyde measured in $\mathrm{CDCl}_{3}$. Additional information such as the generated peak lists or the information on the structure of the assigned sample is shown in the right information panel. The main toolbar for basic edit functions is given above the spectra visualization panel

and intensity of the signal. The example shown in Fig. 3 was gained with the file format RAW (recorded with the ThermoFisher instrument QExactive Plus) demonstrating that the editor can be adapted to read and process also proprietary file formats. With respect to the FAIR data principles, data storage and further processing of proprietary file formats is not a preferred or desired procedure but in some cases, alternatives to the use of proprietary files are currently missing. Therefore, spectra editors should offer options to cover this need if possible. ChemSpectra was used to read data files of two instruments of ThermoFisher [model QExactive Plus (ESI) and Thermo Finnigan Mat 95 (EI and FAB mode)] as test-cases for data that are not given in an open file format. Since the RAW file format contains binary data, it has to be decoded before the processing with ChemSpecta is possible. For this purpose, msConvert in Proteowizard [29] is employed to convert MS files from RAW to mzML. 


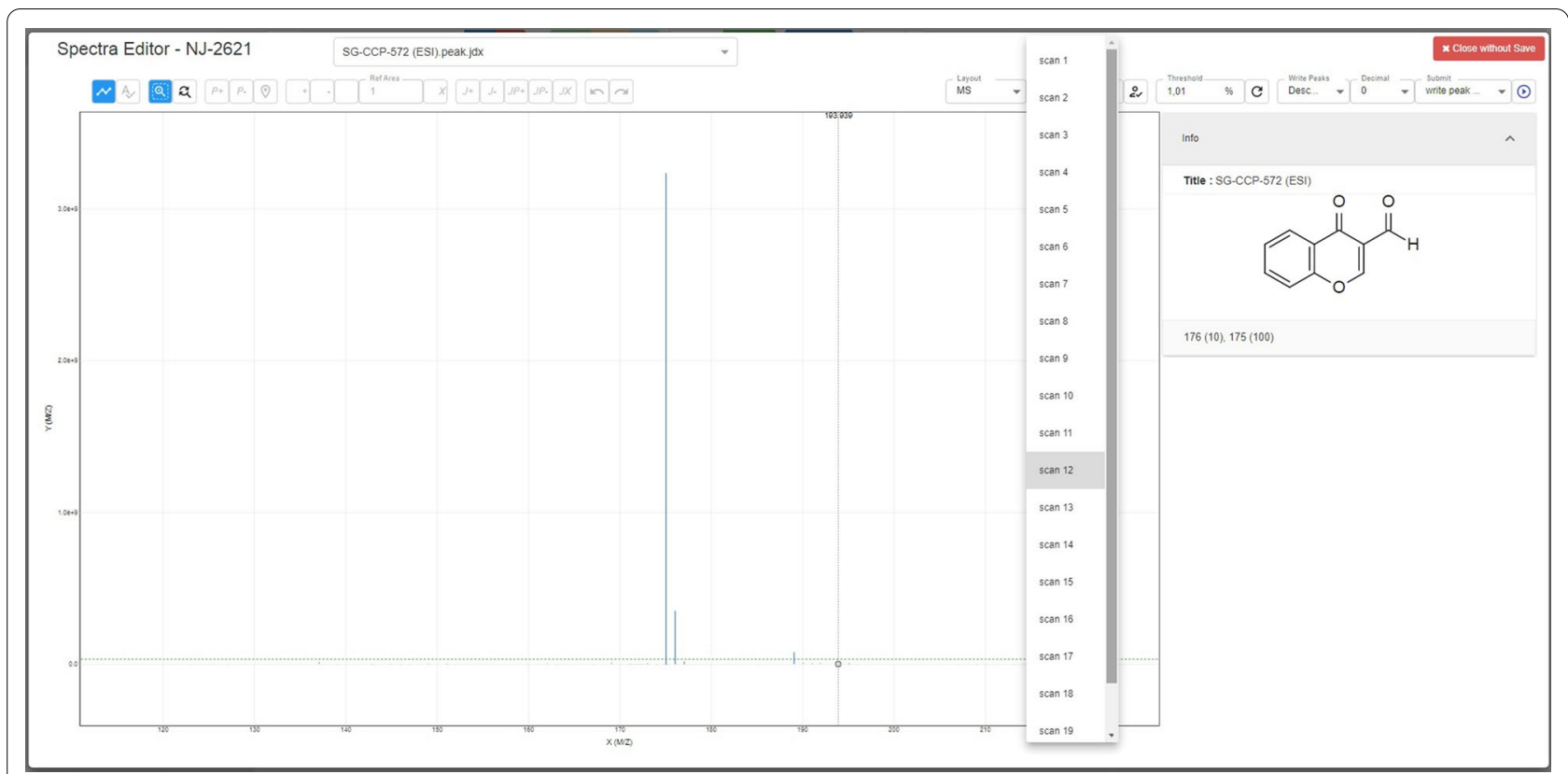

Fig. 3 Overview of the react-spectra-editor for mass spectrometry data applied to the file format RAW that was recorded with a ThermoFisher Modell QExactive Plus mass spectrometer for commercial 4-oxo-4H-chromene-3-carbaldehyde. The selection options for the available scans are shown

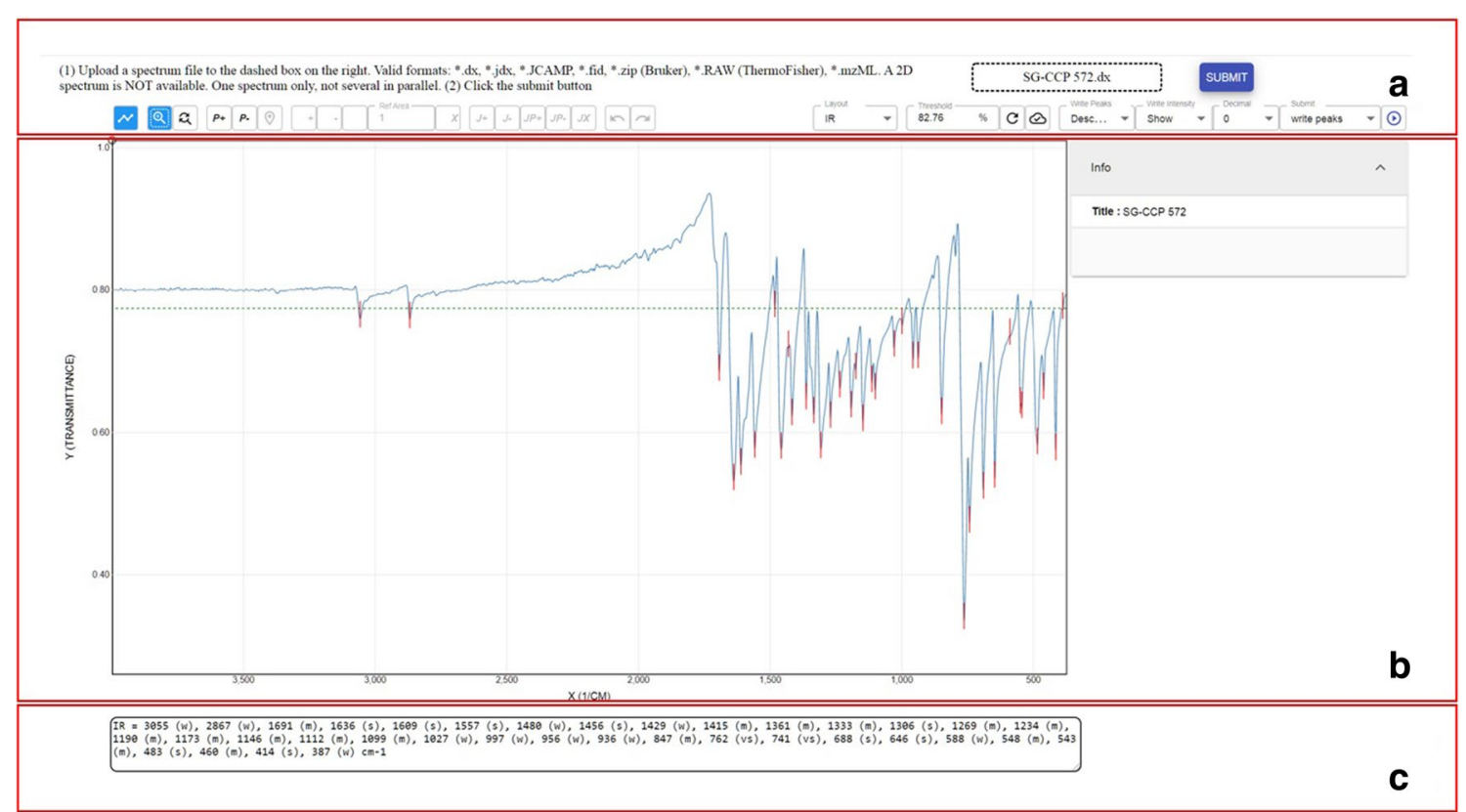

Fig. 4 The ChemSpectra UI as the stand-alone version used for the visualization of an IR spectrum of commercial 4-oxo-4H-chromene-3-ca rbaldehyde, uploaded as JCAMP-DX (*.dx) file. Highlighted are the UI parts a file upload and user input panel for the analysis of spectra, $\mathbf{b}$ react-spectra-editor and additional information to view the results and $\mathbf{c}$ text output panel. The notifications are not shown 
MsConvert in a docker container is called by the chemspectra-app to achieve this job. mzML files are converted to JCAMP-DX using pymzML, an Open Source python mass spectrometry file parser.

\section{Stand-alone version}

In its stand-alone version, ChemSpectra runs with an additional UI-the chem-spectra-client-that allows the user to add input or retrieve output as an alternative to a connection of other resources or target systems. The chem-spectra-client UI provides three functions in addition to the react-spectra-editor: (1) a file management to upload the data to be visualized/analyzed, (2) notifications to the user and (3) a text output that is generated to be copied for further use of the generated data (Fig. 4).

\section{Embedded implementation}

Depending on the desired interactions, the implementation with another web application such as an ELN or repository requires additional efforts for system-specific adaptations. For an implementation with Chemotion ELN, which is described here exemplarily, different work processes of the ELN have to be merged with ChemSpectra including the direct use of files that were generated from analytical instruments. In addition, challenges such as data persistence, supporting the storage of data for the full data life cycle, and a workflow management need to be considered. In this regard, embedding ChemSpectra into the Chemotion ELN was realized by keeping the original input files and adding the newly composed files as persisted data. The original files stored without any modifications are an important resource for any future referencing issues, while the composed files are kept to avoid the need for repeated analyses. Additionally, two images are generated: a low-resolution thumbnail for preview and a higher resolution for the reuse for example in publications. Both images are regenerated every time a user edits a spectrum. The embedding of the ChemSpectra editor results in the availability of a set of file formats that can be generated fully automatically without the input of the user or edited if further actions are desired. Figure 5 shows with the example of an ${ }^{1} \mathrm{H}$ NMR spectroscopy file, how the implementation with the analysis section of Chemotion ELN is realized, giving three relevant files for the user: an original file (*.zip), a user-edited version (".edit.jdx) and an image file (".edit.png) [30]. A direct benefit of the implementation of ChemSpectra with an ELN is the transfer of the gained data analysis to the ELN. This allows the fast analysis of spectroscopic data and the fast and error-free documentation of the obtained results.

\section{Discussion of the limitations of the current developments}

ChemSpectra was developed as a basis to reach independence from commercial software for standard analytical measurements. The software allows the integration with other web-developments and offers flexibility to cover further analytical techniques with forthcoming extensions. In the current version, the focus of the

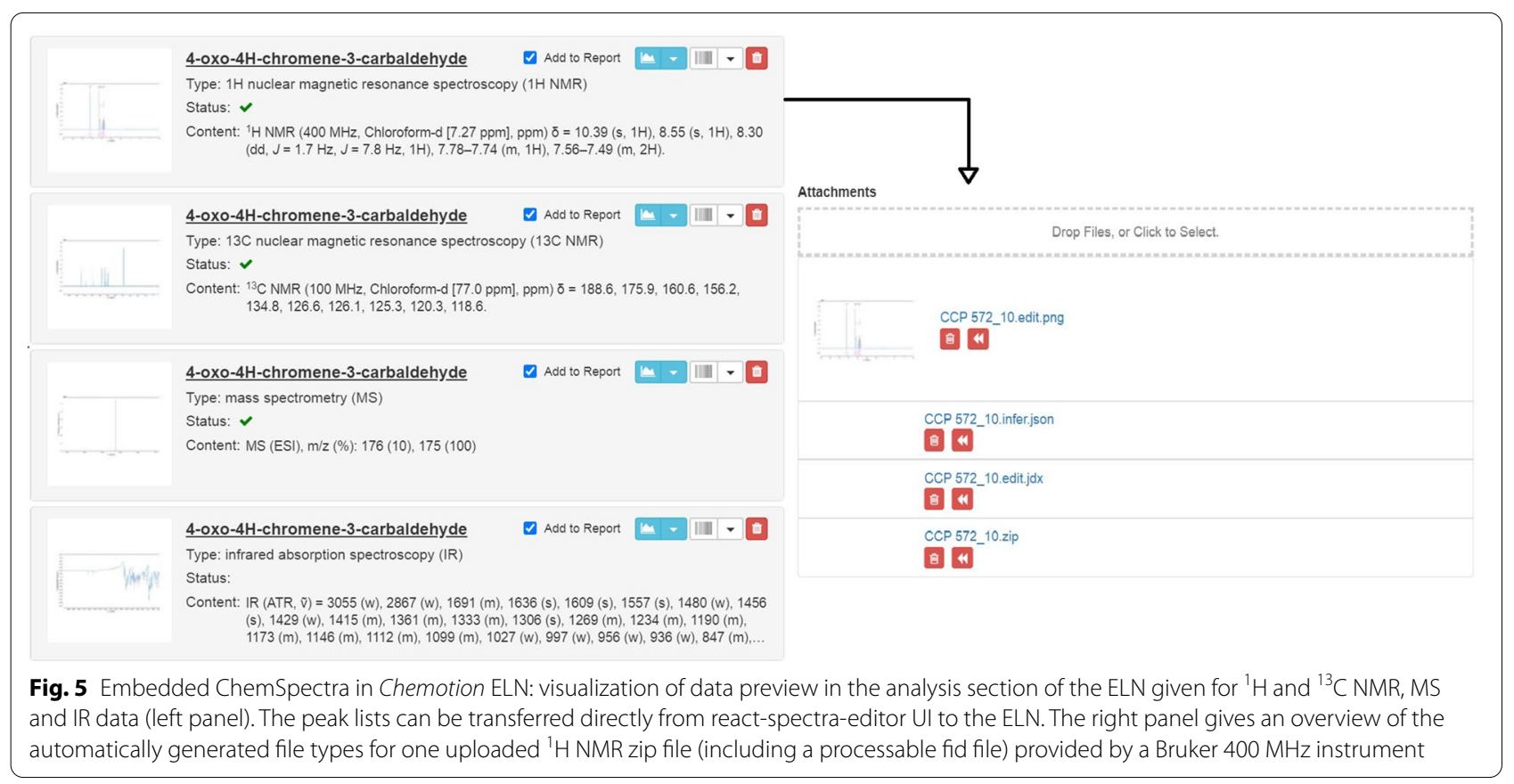


developments lay on the definition of basic functionality that covers the most important needs for NMR, IR and MS spectra analysis and the design of a model for smart integration to web-based information systems. The software does not offer a comprehensive solution to special types of measurement yet and lacks certain functions compared to established and specialized software. Considering for example NMR analyses in Organic Chemistry, further improvements of the editor should contain functions for the viewing and analysis of $2 \mathrm{D}$ data and functions for the comparison of different spectra in one application window. Additionally, the processing of FID files has to be improved by adding advanced phase correction and baseline correction methods (see Additional files 1 for an example). For mass spectrometry analysis, the chem-spectra-app should be extended to support further MS file formats. Initiatives like OpenChrom [31] show how extensive but also successful such a project is when the given challenges are to be solved by the community. The compatibility of ChemSpectra with additional file types and also types of analytical measurements will be an important extension of ChemSpectra in the future, building a framework for interoperable analytical data and its use in full compliance to FAIR principles.

\section{Conclusion}

ChemSpectra is a software to swiftly visualize and analyze analytical data, integrating solutions for IR (infrared spectroscopy), mass spectrometry (MS), and one-dimensional ${ }^{1} \mathrm{H}$ and ${ }^{13} \mathrm{C}$ NMR (proton and carbon nuclear magnetic resonance) spectroscopy data. It serves as a decentralized work-instrument for the analysis of the most often used types of spectroscopic data in synthetic (organic) chemistry research, being able to deal with the open file formats JCAMP-DX (IR, NMR, MS) and mzML (MS). The software is offered as an Open Source to allow the further extension to other file formats by the community as exemplarily shown for mass spectra files of the type RAW and NMR spectroscopy files of type FID gained from common analytical instruments. All data files that are provided as non-JCAMP-DX files are processed and converted to JCAMP-DX, allowing a standardized treatment of all data files after a first processing step. ChemSpectra is provided in two versions, as a standalone version to be used as an independent service and as an integrated editor for the Chemotion web applications electronic lab notebook (ELN) and repository. The embedded ChemSpectra editor allows the storage of the original spectra along with edited versions, the automatic peak detection according to a default or manually defined threshold and the storage of an automatically generated image of the spectra in png format. To maximize the benefit of the embedded editor for users, a workflow to write the automatically detected or manually chosen signals was implemented. This allows the direct transfer of information to e.g. the ELN or repository. ChemSpectra consists of different modules that are used to build the core software (chem-spectra-app and react-spectraeditor) and the necessary extensions for its use as standalone service (chem-spectra-client). As exemplified with the Chemotion ELN and repository implementation, it can be adapted to other work environments. ChemSpectra should serve as a basic software to be extended in the future with respect to further data type-specific analysis functions and its usability for additional file formats. ChemSpectra is released under the AGPL license to encourage its re-use and further developments by the community.

\section{Supplementary Information}

The online version contains supplementary material available at https://doi. org/10.1186/s13321-020-00481-0.

Additional file 1. The Supporting Information contains further documentation on the transformation of spectra with ChemSpectra, Flow charts for processing spectra of different file formats, decoding and parsing of mass spec, NMR and IR data files. Also, the communication overview with the ELN environment is given for the ELNembedded ChemSpectra software. Examples for processing of JCAMP-dx files and fid files in comparison are given.

\section{Abbreviations}

ELN: Electronic Laboratory Notebook; UI: User interface; JCAMP-DX: Joint Committee on Atomic and Molecular Physical data extension; NMR: Nuclear magnetic resonance; IR: Infrared; MS: Mass spectrometry; AGPL: Affero General Public License.

\section{Acknowledgements}

We acknowledge the support by Deutsche Forschungsgemeinschaft and Open Access Publishing Fund of Karlsruhe Institute of Technology. This work was supported by the Helmholtz program Biointerfaces in Technology and Medicine (BIFTM) and by bwUniCluster, bwFORCluster. For computational resources we acknowledge the bwCloud (https://www.bw-cloud.org), funded by the Ministry of Science, Research and Arts Baden-Württemberg (Ministerium für Wissenschaft, Forschung und Kunst Baden-Württemberg).

\section{Authors' contributions}

YCH developed the herein described work concerning frontend and backend developments and adapted the ChemSpectra software to the additional challenges that were provided by the corresponding authors. PT and AN added valuable input and suggestions in discussions. NJ and SB supervised the development and supported with the interpretation of chemistry specific problems. All authors read and approved the final manuscript.

\section{Funding}

Open Access funding enabled and organized by Projekt DEAL. This project has been funded by the German Research Foundation (Deutsche Forschungsgemeinschaft, 266379491) and the Ministry of Science, Research and Arts BadenWürttemberg (Ministerium für Wissenschaft, Forschung und Kunst BadenWürttemberg) through the Science Data Center MoMaF. We acknowledge the support of the VirtMat research consortium at the KIT.

\section{Availability of data and materials}

The Additional file contains further documentation on the transformation of spectra with ChemSpectra, Flow charts for processing spectra of different file formats, decoding and parsing of mass spec, NMR and IR data files. Also, 
the communication overview with the ELN environment is given for the ELN-embedded ChemSpectra software. Examples for processing of JCAMP$\mathrm{dx}$ files and fid files in comparison are given. Project name: ChemSpectra. Project homepage: eln.chemotion.net. Project demo page: https://eln.chemo tion.net/chemspectra-editor. Additional videos are deposited here: https:// github.com/ComPlat/react-spectra-editor. Operating system(s): platformindependent access, developed/tested on Linux and Mac, deployed on Linux. Library requirements (for developer): (1) user interface: react-spectra-editor (dependencies: React.js [32]; jcampconverter [33], D3.js [34] (jcamp processing in JavaScript). (2) Peak-picking and jcamp processing: chem-spectra-app (and dependencies: flask (web server) [35], nmrglue (spectra reading) [36], pymzML [37] (mzML to JCAMP-DX conversion), pyopenms [38] (mzXML to JCAMP-DX conversion), netCDF4 [39] (cdf to JCAMP-DX conversion), matplotlib (image processing), numpy [40], and scipy (peak-picking) [41]. (3) Coupling constant and multiplicity inference: cheminfo-js/spectra [30]. (4) RAW to mzML conversion: docker and msConvert in Proteowizard [29], numpy [32], scipy [19, 41] and Matplotlib [42]. Other requirements: Modern internet browser supporting HTML5 and JavaScript. Recommended browsers: Chrome. Programming language: JavaScript, Python. Source Code on Github: Chem-spectra-app (https ://github.com/ComPlat/chem-spectra-app) chem-spectra-client (https://githu b.com/ComPlat/chem-spectra-client), and react-spectra-including demos and videos (https://github.com/ComPlat/react-spectra-editor) can be retrieved from github. Source Code on Zenodo: chem-spectra-client: https://doi. org/10.5281/zenodo.4059273; react-spectra-editor https://doi.org/10.5281/ zenodo.4059278; chem-spectra-app https://doi.org/10.5281/zenodo.4059263. License: AGPLv3.

\section{Ethics approval and consent to participate}

Not applicable.

\section{Consent for publication}

Not applicable.

\section{Competing interests}

The authors declare that they have no competing interests.

\section{Author details}

${ }^{1}$ Institute of Biological and Chemical Systems-Functional Molecular Systems (IBCS-FMS), Karlsruhe Institute of Technology, Hermann-von-Helmholtz-Platz 1, 76344 Eggenstein-Leopoldshafen, Germany. ${ }^{2}$ Institute of Organic Chemistry, Karlsruhe Institute of Technology, Fritz-Haber-Weg 6, 76131 Karlsruhe, Germany.

Received: 15 July 2020 Accepted: 15 December 2020

Published online: 10 February 2021

\section{References}

1. https://doi.org/10.1021/ja906709t. Accessed 14 Nov 2019

2. https://docs.chemaxon.com/display/docs/NMR+Predictor\#NMRPredict or-NMRSpectrumPreviewPanel. Accessed 13 Nov 2019

3. Link to free TopSpin download. https://www.bruker.com/service/suppo rt-upgrades/software-downloads/nmr/free-topspin-processing/nmrtopspin-license-for-academia.html

4. https://www.effemm2.de/spectragryph/. Accessed 13 Nov 2019

5. Lancashire RJ (2007) The JSpecView Project: an Open Source Java viewer and converter for JCAMP-DX, and XML spectral data files. Chem Cent J $1: 31$

6. http://wwwchem.uwimona.edu.jm/software/jcampdx.html. Accessed 13 Nov 2019

7. Mohamed A, Nguyen CH, Mamitsuka H (2016) NMRPro: an integrated web component for interactive processing and visualization of NMR spectra. Bioinformatics 32:2067-2068

8. Xia J, Mandal R, Sinelnikov IV, Broadhurst D, Wishart DS (2012) MetaboAnalyst 2.0-a comprehensive server for metabolomic data analysis. Nucleic Acids Res 40:W127-W133

9. Tulpan D, Léger S, Belliveau L, Culf A, Čuperlović-Culf M (2011) MetaboHunter: an automatic approach for identification of metabolites from 1H-NMR spectra of complex mixtures. BMC Bioinf 12:400
10. Zhang F, Brüschweiler R (2007) Robust deconvolution of complex mixtures by covariance TOCSY spectroscopy. Angew Chem Int Ed 46:2639-2642

11. Vosegaard T (2015) jsNMR: an embedded platform-independent NMR spectrum viewer. Magn Reson Chem 53:285-290

12. Beisken S, Conesa P, Haug K, Salek RM, Steinbeck C (2015) SpeckTackle: JavaScript charts for spectroscopy. J Cheminform 7:17

13. https://www.ebi.ac.uk/metabolights/index

14. Wishart DS, Jewison T, Guo AC et al (2012) HMDB 3.0 - the human metabolome database in 2013. Nucleic Acids Res 41:D801-D807

15. https://www.drugbank.ca/. Accessed 14 Nov 2019

16. Wishart DS, Feunang YD, Guo AC et al (2017) DrugBank 5.0: a major update to the DrugBank database for 2018. Nucleic Acids Res 46(D1):D1074-D1082

17. https://github.com/cheminfo-js/jcampconverter\#readme. Accessed 13 Nov 2019

18. https://github.com/ComPlat/nmrglue/commits/show-all-data. Accessed 13 Nov 2019

19. Virtanen P, Gommers R, Oliphant TE, Haberland M, Reddy T, Cournapeau D, Burovski E, Peterson P, Weckesser W, Bright J, van der Walt SJ, Brett M, Wilson J, Millman KJ, Mayorov N, Nelson ARJ, Jones E, Kern R, Larson E, Carey CJ, Polat I, Feng Y, Moore EW, VanderPlas J, Laxalde D, Perktold J, Cimrman R, Henriksen I, Quintero EA, Harris CR, Archibald AM, Ribeiro AH, Pedregosa F, van Mulbregt P, SciPy 1.0 Contributors. (2019) SciPy 1.0_ fundamental algorithms for scientific computing in Python. Preprint arXiv :1907.10121

20. Tremouilhac $P$, Nguyen A, Huang Y-C, Kotov S, Lütjohann DS, Hübsch F, Jung N, Bräse S (2017) Chemotion ELN: an Open Source electronic lab notebook for chemists in academia. J Cheminform 9:54

21. Tremouilhac P, Lin C-L, Huang P-C, Huang Y-C, Nguyen A, Jung N, Bach F, Neumair B, Streit A, Bräse S (2020) The repository chemotion: infrastructure for sustainable research in chemistry. ChemRxiv. https://doi. org/10.26434/chemrxiv.12195318.v1

22. Potthoff J, Tremouilhac P, Hodapp P, Neumair B, Bräse S, Jung N (2019) Procedures for systematic capture and management of analytical data in academia. Anal Chim Acta 1:100007

23. https://github.com/ComPlat/chem-spectra-app. Accessed 13 Nov 2019

24. https://github.com/ComPlat/chem-spectra-client. Accessed 13 Nov 2019

25. https://github.com/ComPlat/react-spectra-editor. Accessed 13 Nov 2019

26. Deutsch EW (2010) Mass spectrometer output file format mzML. Methods Mol Biol 604:319-331

27. https://github.com/cheminfo-js/spectra

28. Cobas JC, Constantino-Castillo V, Martín-Pastor M, del Río-Portilla F (2005) A two-stage approach to automatic determination of $1 \mathrm{H}$ NMR coupling constants. Magn Reson Chem 43(10):843-848. https://doi.org/10.1002/ mrc.1623

29. Chambers MC, MacLean B, Burke R, Amode D, Ruderman DL, Neumann S, Gatto L, Fischer B, Pratt B, Egertson J, Hoff K, Kessner D, Tasman N, Shulman N, Frewen B, Baker TA, Brusniak MY, Paulse C, Creasy D, Flashner L, Kani K, Moulding C, Seymour SL, Nuwaysir LM, Lefebvre B, Kuhlmann F, Roark J, Rainer P, Detlev S, Hemenway T, Huhmer A, Langridge J, Connolly B, Chadick T, Holly K, Eckels J, Deutsch EW, Moritz RL, Katz JE, Agus DB, MacCoss M, Tabb DL, Mallick P (2012) A cross-platform toolkit for mass spectrometry and proteomics. Nat Biotechnol 30:918-920

30. A forth file format, ${ }^{*}$.infer.json, is generated for internal processes and data comparison.

31. https://lablicate.com/platform/openchrom. Accessed 14 Nov 2019

32. https://github.com/facebook/react/. Accessed 13 Nov 2019

33. https://github.com/cheminfo-js/jcampconverter. Accessed 14 Nov 2019

34. https://github.com/d3/d3. Accessed 19 Dec 2020

35. https://github.com/pallets/flask. Accessed 13 Nov 2019

36. https://github.com/jjhelmus/nmrglue. Accessed 13 Nov 2019

37. Kösters M, Leufken J, Schulze S, Sugimoto K, Klein J, Zahedi RP, Hippler M, Leidel SA, Fufezan C (2018) pymzML v2.0: introducing a highly compressed and seekable gzip format. Bioinformatics 34:2513-2514

38. Röst HL, Schmitt U, Aebersold R, Malmström L (2014) pyOpenMS: a Python-based interface to the OpenMS mass-spectrometry algorithm library. Proteomics 14(1):74-77. https://doi.org/10.1002/pmic.201300246

39. https://github.com/Unidata/netcdf4-python. Accessed 19 Dec 2020

40. https://www.numpy.org/. Accessed 13 Nov 2019

41. https://github.com/scipy/scipy. Accessed 13 Nov 2019 
42. https://matplotlib.org/. Accessed 13 Nov 2019

\section{Publisher's Note}

Springer Nature remains neutral with regard to jurisdictional claims in published maps and institutional affiliations.

- fast, convenient online submission

- thorough peer review by experienced researchers in your field

- rapid publication on acceptance

- support for research data, including large and complex data types

- gold Open Access which fosters wider collaboration and increased citations

- maximum visibility for your research: over 100M website views per year

At BMC, research is always in progress.

Learn more biomedcentral.com/submissions 